\title{
The Analysis of China's Grain Output Fluctuation Based on EMD
}

\author{
Qiting Chen ${ }^{1} \&$ Meng Wang ${ }^{1}$ \\ ${ }^{1}$ School of Economics and Management, Nanjing University of Aeronautics and Astronautics, Nanjing, China \\ Correspondence: Meng Wang, School of Economics and Management, Nanjing University of Aeronautics and \\ Astronautics, Nanjing, China. Tel: 86-156-0518-9830. E-mail: 15605189830@163.com
}

Received: September 2, 2017

Accepted: September 22, 2017

Online Published: October 1, 2017

doi:10.5539/ijef.v9n11p64

URL: https://doi.org/10.5539/ijef.v9n11p64

\begin{abstract}
Food is one of the most important resources for staying alive. This paper analyzes grain output fluctuations and their driving forces in China from 1978 to 2014, based on Empirical Mode Decomposition (EMD) method. These results show that there are two type cycles of cyclical fluctuation, one is 3-yearterm, and another is 8-year term. These results show that the 8-year cyclical fluctuation is the major term. Grain production's cyclical fluctuation in 3 years was mainly influenced by yield of grain per unit area from 1978-2004 and 2007-2014, and by the area sown from 2004 to 2007. On the other hand, the longer cyclical fluctuation of 8 years is mainly affected by the yield of grain per unit area. The grain output is predicted for the next three years through the RBF neural network optimized by PSO. These results show that China's annul grain output in the next three years will be stabilized at about 600 million tons, which may grow slowly though.
\end{abstract}

Keywords: grain output, fluctuation, EMD, RBF

\section{Introduction}

As one of the most important resource, food is a key factor that affects national and regional social stability. Growing enough food is particularly important for China, because China is the world's most populous country, which feeds $18.84 \%$ of the world's population. Food production directly affects people's life and national stability. So, this research is indispensable for keeping food production stable. In numerous studies of food production, studies about the formation of grain output fluctuations are beneficial to adopt effective measures about stabilize grain production, reducing the fluctuation intensity. Forecasting of grain production accurately is conducive to take corresponding measures to expand or control food production, and to achieve food safety production as well.

At present, the researchers have done a lot of efforts on grain production fluctuation, the past research has focused on the short cycle. In recent years, the research of the long period of food production gradually becomes mainstream direction. In past studies, many traditional methods like residual method, quarter-on-quarter growth method, etc., were used to study short cycle fluctuation of food production. In recent years, in order to study further on the long period of grain production fluctuation, the researchers have tried and made a lot of progress in Fourier analysis and wavelet analysis, the HP filter, empirical mode decomposition method, etc. However, these studies found out that although the Fourier analysis method can obtain higher resolution in frequency dominion, but powerless in the time domain especially for nonlinear and non-stationary data. Although wavelet analysis can keep time domain characteristics of signal and has a certain frequency resolution at the same time, but the improvement of accuracy of time domain and frequency domain is restricted and will produce a lot of false harmonic at the same time because of the uncertainty principle. When the Empirical Mode Decomposition (EMD) method take forward by N. E. Huang in 1998 overcome the shortcomings above, and it can be used to analyze nonlinear data well. Considering that all data analyzed are nonlinear, this paper will analyze grain output by EMD method.

More and more researchers now use the EMD method to study nonlinear data. Some of them have used the EMD to analyze the grain output, and have obtained reasonable results. Liu et al analyzed the grain production fluctuation in China between 1948 and 2003 using EMD. According their research, China's grain output fluctuations had two cycles, respectively were short cycle fluctuation in 3-years and the 9-years long cycle, and they thought that the food production in China in 2005 or 2006 would be in serious shortages according to the shape of the IMF function curve. Li et al analyzed the grain output in Hebei province from 1978 to 2005 using 
EMD and found that grain output in Hebei province also existed quasi-periodic fluctuation, and there were three cycles, respectively for 4 years term, 6 years term and 11 years term. There are more and more application of EMD in food production fluctuation analysis, and this paper will analyze China's grain output fluctuation and the cause from 1978 to 2014 through EMD decomposition, at the last, set up a Radial Basis Function (RBF) neural network optimized by Particle Swarm Optimization (PSO) to predict the grain output in China in the next three years.

\section{Methods}

\subsection{EMD}

Empirical mode decomposition (EMD) was a new method of signal processing put forward by N. E. Huang in the national aeronautics and space administration (NASA) in 1998, it is direct, intuitive, and based on experience and adaptive, it is a good method to deal with nonlinear and non-stationary signal. The EMD method decomposes a time domain signal into multiple vector by "filtering" step by step, and then results in the Intrinsic Mode Function (IMF) as well as a trend vector. There are two "filter" conditions: 1) the number of poles and zeros are equal or differ at most 1 ; 2) the mean value of the upper and lower envelope determined by its maximum value and minimum value is zero at any point. The EMD method "filter" out each IMF one by one from the time domain signal by the "filter" conditions and then the trend vector is the last remaining. The specific steps of EMD are as the following flow chart:

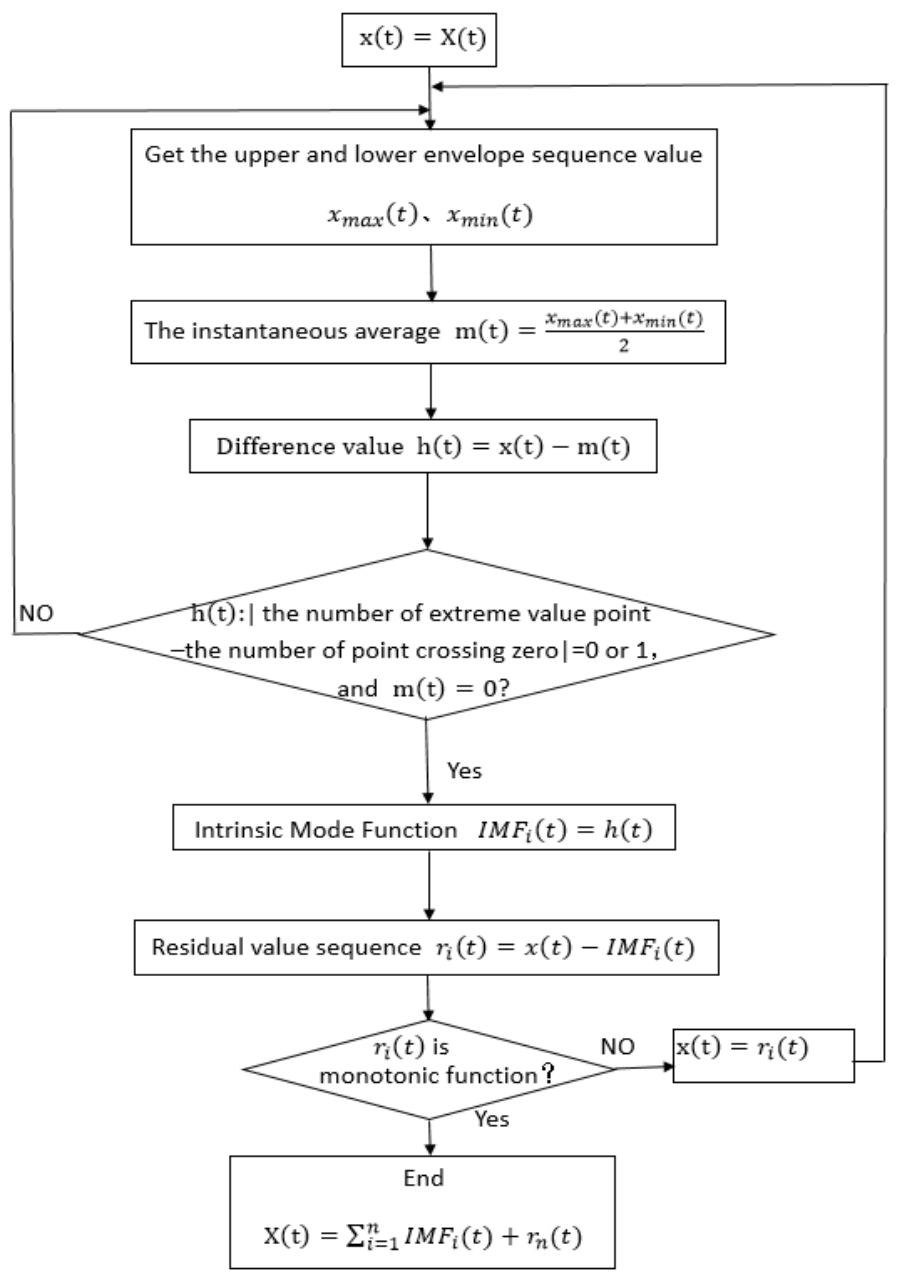

Figure 1. The flow chart of the EMD decomposition

\subsection{RBF Neural Network}

Radial Basis Function (RBF) neural network is a kind of feed forward neural networks, which includes input layer, hidden layer and output layer. The input layer, the first one, is composed of signal source node. The second 
is the hidden layer, wherethe transformation function of neurons is radial basis function. It is a partial response function, and the number of nodes in hidden layer is determined by the specific situation. The third layer is output layer, which is a response to the input.

RBF neural network can approximate any nonlinear function, and because of its good generalization ability and fast convergent speed, it is widely used in pattern recognition, information processing, nonlinear function approximation, time series analysis, system modeling, control and fault diagnosis, image processing, and so on.

\section{Fluctuation of Grain Output and Its Cause Analysis}

\subsection{Variable Selection and Data Sources}

These data used in this study include total grain yield, grain yield and sown area of grain in China from 1978 to 2014. All the research data in this paper are from the "China Statistical Yearbook" in National Statistical Bureau, and all the data processing are completed on the R2014b MATLAB software platform.

\subsection{Fluctuation Analysis of Grain Output in China}

Figure 2 is China's grain output curve from 1978 to 2014, from Figure 2, we can see that, China's grain output has been in fluctuation, but overall, it is on the rise since 1978.

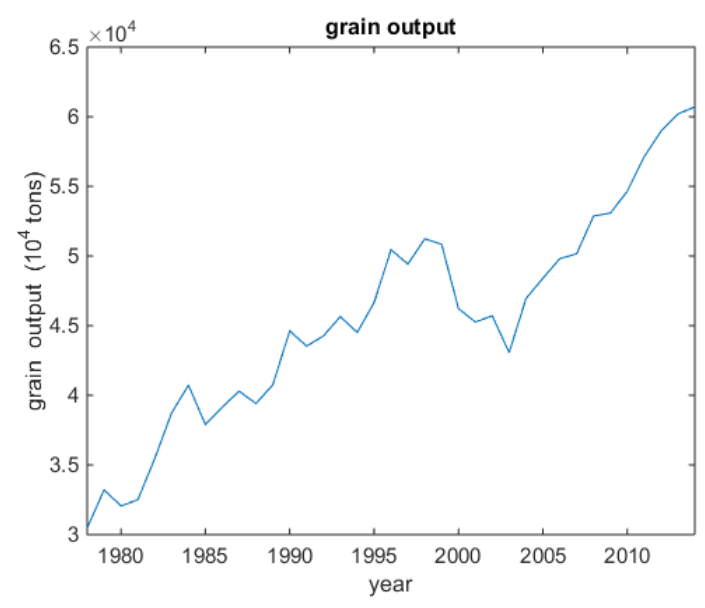

Figure 2. Grain output

Figure 3 is the EMD results of China's grain production from 1978 to 2014, including 2 IMF components and 1 trend component $\mathrm{R}$, and Table 1 is their respective variance contribution rate.
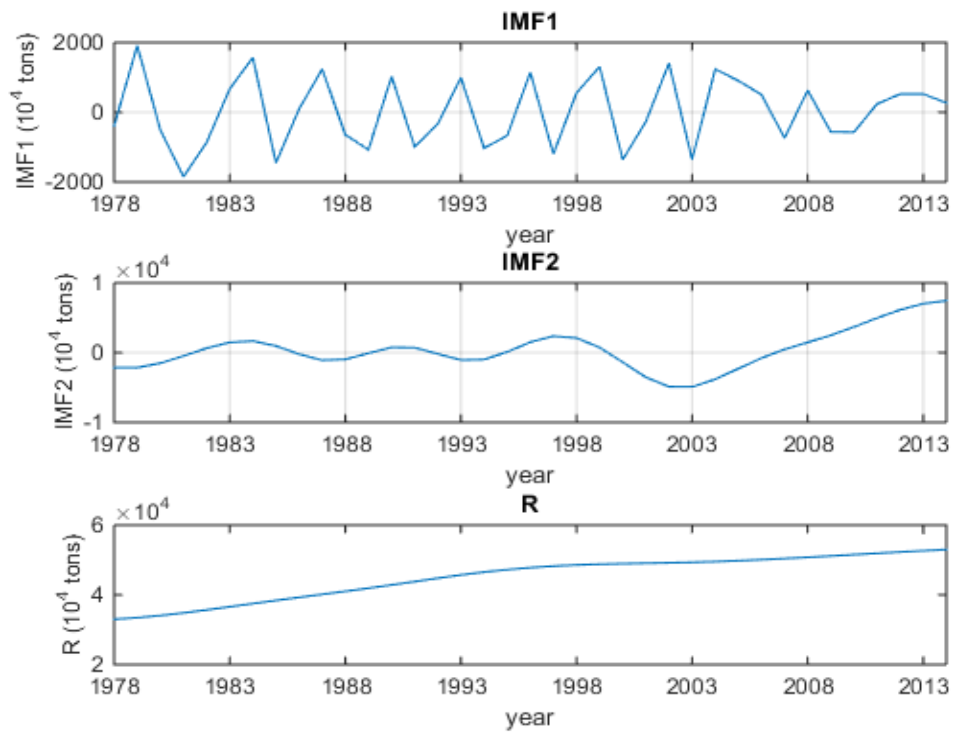

Figure 3. The EMD results of grain output 
Table 1. Variance contribution rates of grain output's EMD results

\begin{tabular}{lccc}
\hline & IMF1(3years) & IMF2(8years) & $\mathrm{R}$ \\
\hline Variance contribution rate & $2.09 \%$ & $17.38 \%$ & $80.53 \%$ \\
\hline
\end{tabular}

In Figure 3, the curve of trend term $\mathrm{R}$ is monotonically increasing, which indicates that the grain yield is increasing continuously. The fluctuation of IMF1 curve generally shows 11 cycles, and the average cycle is 3 years, indicating that there is a periodic fluctuation of grain output in China for a period of 3 years, while the fluctuation of IMF2 curve shows 3.5 cycles, but average cycle is 8 years, suggesting that there is also a cyclical fluctuations of China's food production for quasi 8 years. From table 1, although the trend $\mathrm{R}$ has the largest variance contribution rate as $80.53 \%$, the variance contribution rate of the IMF2 is pretty large as $17.3 \%$, suggesting that the fluctuation of grain output in the period of 8 years will have great influence on the stability of grain production, grain supply and demand in China. But even though the variance contribution rate of IMF1 is only $2.09 \%$ as the minimum, considering that China is the biggest country with large population and grain output, quasi 3 years cycle can also not be ignored, and should attract enough attention of relevant government departments while making the strategic grain reserves planning.

\subsection{China's Grain Output Fluctuation Reason Analysis}

While many factors influence the grain output, such as technology advances, agricultural machinery total power, effective irrigation area, the affected area and so on, the impact of these factors on grain output is finally shown on the sown area of grain and unit area yield of grain, whicht are the most direct factors affecting grain output.

In order to analyze the cause of China's grain output fluctuation further, the EMD has been done on the planting area sequences and yield sequences for that the planting area and unit area yield of grain are the most direct factors affecting food production, and then respectively compared to the EMD results of grain output sequence. And according to the dimension of each are different, the IMF components of these three variables are normalized primarily, and then after the normalization, the IMF1 of the fluctuation of grain production in China were compared respectively with the IMF1 of the unit area yield of grain and the IMF1 of the sown area of grain, as shown in Figure 4 and Figure 5.

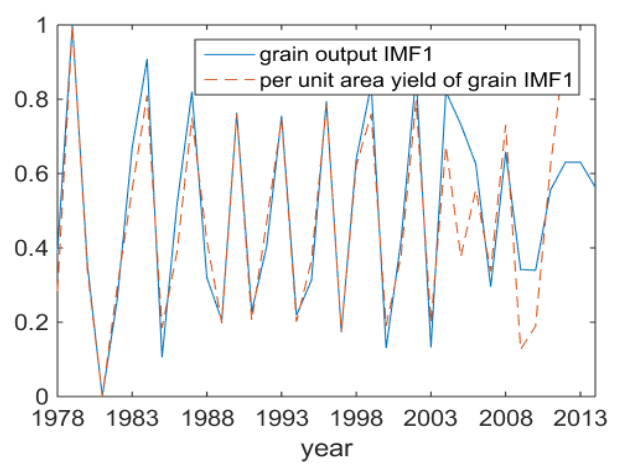

Figure 4. Comparison between IMF1 (grain output) and IMF1 (unit area yield of grain)

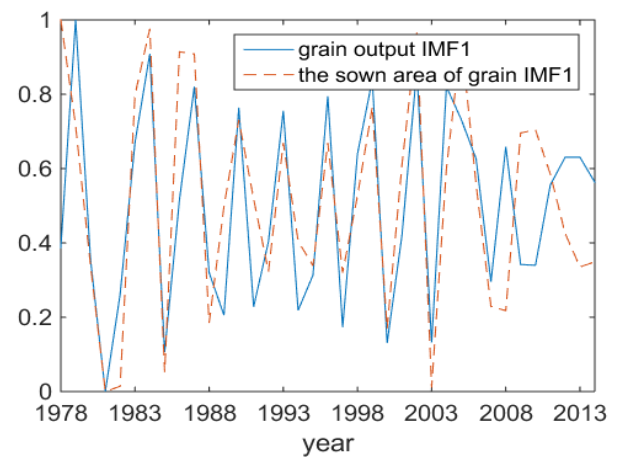

Figure 5. Comparison between IMF1 (grain output) and IMF1 (the sown area of grain) 
As Figure 4 showing, in addition to the waveform is not consistent between 2004 and 2007, the IMF1 curve of grain output fluctuation and the IMF1 curve of unit area yield of grain are basic synchronous, their peaks and troughs are almost consistent. According to Figure 5, the IMF1 curve of grain output fluctuation and the IMF1 curve of the area sown are basic consistent from 2004 to 2007, but inconsistent since 2007, and though the wave direction of these two curve are the same before 2004, the alignment is lower compared with the Figure 4. Therefore, this paper argues that between 1978 and 2004, the short 3 years cyclical fluctuations of food output was mainly controlled by unit area yield of grain although this periodic fluctuation was influenced by the area sown too, and between 2004 and 2007, the area sown became the main influence factors of the short 3 years cyclical fluctuations, but after 2007 till 2014, the quasi 3 years cycle of grain output was controlled again by unit area yield of grain.

Figure 6 is comparison between the IMF2 of grain output in China and the IMF2 of area sown after normalized. Figure 7 is comparison between the IMF2 of China's grain output and the IMF3 of area sown after normalized, and Figure 8 is the comparison between the IMF2 of China's grain output and the IMF2 of unit area yield of grain.

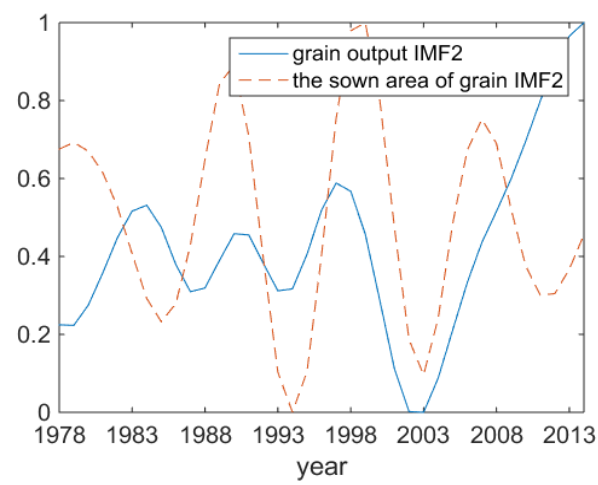

Figure 6. Comparison between IMF2 (grain output) and IMF2 (the sown area of grain)

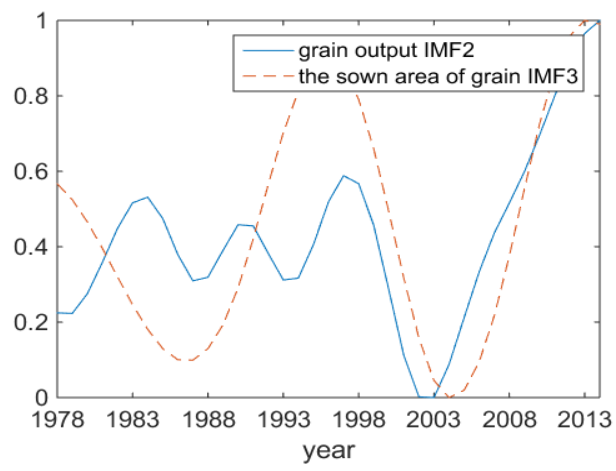

Figure 7. Comparison between IMF2 (grain output) and IMF3 (the sown area of grain)

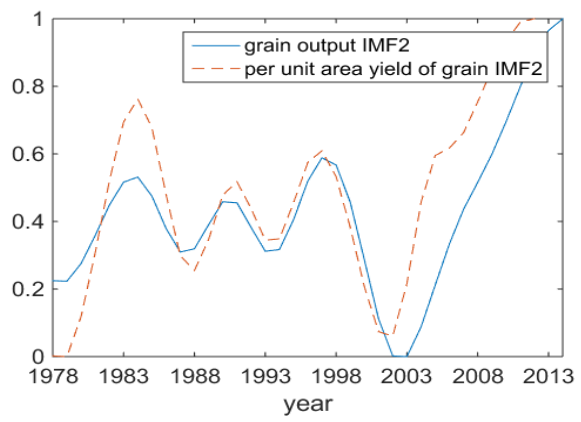

Figure 8. Comparison between IMF2 (grain output) and IMF2 (unit area yield of grain) 
From Figure 8, we can see that the fluctuations of the IMF2 of China's grain output and the IMF2 of unit area yield of grain are basically consistent, the waveform of these two curves are almost the same. Therefore, this paper argues that the 8 years cyclical fluctuations of China's grain output was mainly controlled by unit area yield of grain from 1978 to 2014 .

\section{Prediction of China's Grain Output in the Next Three Years}

\subsection{The RBF Neural Network Prediction Model of Grain Output}

Set existing data of grain output as $\left(x_{1}, x_{2}, \cdots, x_{n}\right)$, this mathematical expression of the RBF neural network model for prediction of grain output is:

$$
x_{n+1}=F\left(x_{n-m}, x_{n-m+1}, \cdots, x_{n}\right)
$$

In the expression above, $\mathrm{m}$ is the optimal order number determined after the extension of order, $x_{n+1}$ means the projections of grain output in the year $\mathrm{n}+1$.

\subsection{Determine the Order of Prediction Model}

Grain production data is a one dimensional time series data, and to determine the order of this prediction model through nonlinear method can get a RBF network model with multiple input and single output, and then we can know the input and output vector of the RBF prediction model according to the expression (1).

$$
X=\left[\begin{array}{cccc}
x_{1} & x_{2} & \cdots & x_{m} \\
x_{2} & x_{3} & \ldots & x_{m+1} \\
\vdots & \vdots & & \vdots \\
x_{n-m} & x_{n-m+1} & \cdots & x_{n-1}
\end{array}\right], Y=\left[\begin{array}{c}
x_{m+1} \\
x_{m+2} \\
\cdots \\
x_{n}
\end{array}\right]
$$

This specific process of the prediction model is shown in Fig. 10, and the root-mean-square-error (RMSE) is used as one of model performance evaluation indicators to measure if this model is good or not. Run the program on the R2014b MATLAB software platform according to the process shown in Fig. 9, the result shows that the optimal order number $\mathrm{m}=7$.

$$
R M S E=\sqrt{\frac{1}{n} \sum_{i=1}^{n}\left(x_{i}-\widehat{x_{l}}\right)^{2}}
$$

In the equation (3), $x_{i}$ represent the actual value of grain output, $\widehat{x}_{\imath}$ represent the predicted value of grain output, and $\mathrm{n}$ represent the sample size for forecasting.

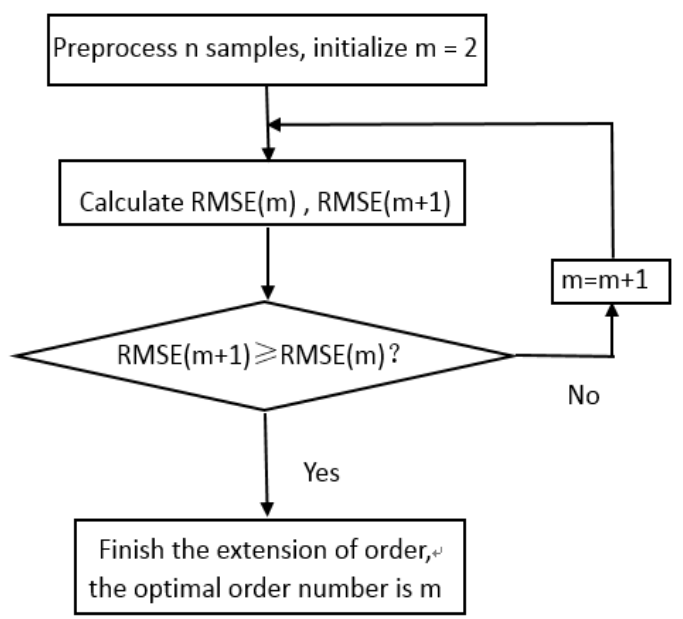

Figure 9. Determination process of the model order

\subsection{Modeling and Validation}

The optimal order number of grain output prediction model determined by nonlinear method is 7 , it means that the first seven years of grain output can be input of RBF neural network and current grain output as output. These data used in this article are grain output in China from 1978 to 2014, 37 years in total, due to optimal order number, the sample size is 30 , and the first 23 samples are used as training data of the network, while the 7 samples behind used as the test data. Optimizing parameters of RBF neural network by Particle Swarm 
Optimization (PSO) method, at the same time, the network is trained by the first 23 samples, and then we can get the RBF neural network model of grain output prediction. Once we have built this model, we can use the model to predict the food production of 7 years from 2008 to 2014 to test its ability to predict. The modeling process is shown in Figure 10.

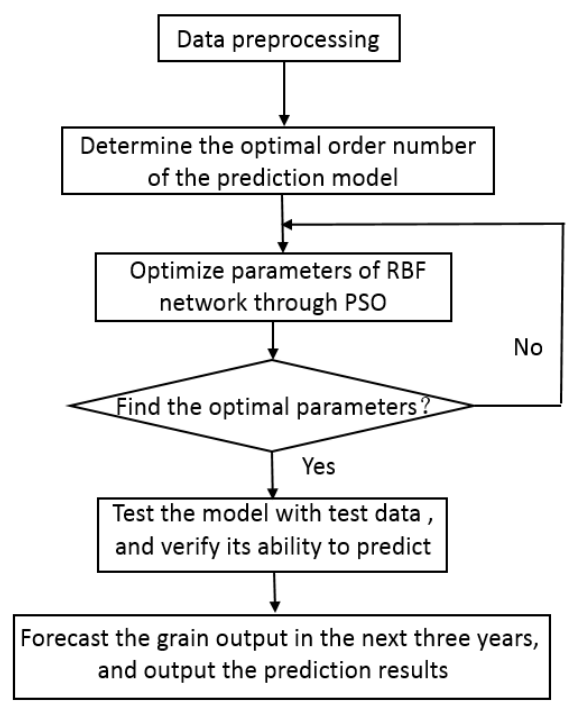

Figure 10. The flow chart of establishing forecast model of

The structure of RBF neural network is 7-3-1. When PSO algorithm is used to optimize the network parameters, the number of iterations $\mathrm{G}=400$, the inertia weight $\mathrm{w}=0.1$, population size is 20 , the acceleration factor c_ $1=$ c $2=2$. Network momentum factor $\alpha=0.15$, the learning rate $\eta=0.8$. Forecast test data with the optimized $\mathrm{RBF}$ neural network model, the prediction results are as shown in table 2.

Table 2. The verification value of grain output from 2008 to 2014

\begin{tabular}{cccc}
\hline Year & Grain output (true value) (million tons) & Prediction of grain output (million tons) & Relative error $(\%)$ \\
\hline 2008 & 52870.92 & 48423.83 & 8.41 \\
2009 & 53082.08 & 49911.68 & 5.97 \\
2010 & 54647.71 & 55123.88 & 0.87 \\
2011 & 57120.85 & 53757.56 & 5.89 \\
2012 & 58957.97 & 55770.89 & 5.41 \\
2013 & 60193.84 & 57020.55 & 5.27 \\
2014 & 60702.61 & 59560.43 & 1.88 \\
MAPE $(\%)$ & & 4.8146 & \\
\hline
\end{tabular}

Relative error $=$ (predicted value - true value $) /$ true value* $100 \%$.

Mean Absolute Percentage Error (MAPE) is used to measure the performance of the prediction model, as shown in table $\Pi$, the RBF neural network model built in this paper can forecast grain output well.

$$
M A P E=\frac{1}{n} \sum\left|\frac{x_{i}-\widehat{x_{l}}}{x_{i}} \times 100\right|
$$

\subsection{The Next Three Years Grain Output Prediction}

Forecast food production in the next three years from 2015 to 2017 using the prediction model established above, forecast results are shown in Table 3.

Table 3. Predicted value of grain output in the next three years

\begin{tabular}{cccc}
\hline Year & 2015 & 2016 & 2017 \\
\hline Grain output prediction (million tons) & 58636.41 & 60075.71 & 61327.70 \\
\hline
\end{tabular}


Though this prediction model still exist some error, but according to the table 3, we can still know that China's grain output in the next three years will remain at around 600 million tons.

\section{Conclusion}

Since 1978, China's grain output was on the rise generally, After EMD analyses, we can know that the grain output fluctuation can be divided into two cycles of cyclical fluctuations, respectively for 3 years and 8 years, and given priority to the periodic fluctuation of 8 years. The short 3-year cyclical fluctuations was mainly controlled by unit area yield of grain from 1978 to 2004 and 2007 to 2014, but it was affected by the area sown from 2004 to 2007 mainly, While the longer cyclical fluctuation of 8 years was mainly controlled by the unit area yield of grain.

According to the results of this article, unit area yield of grain output had a huge impact on food production, but the influence of the area sown can also not be ignored. The relevant departments must take enough attention to these two factors when they make some policies of grain production, consequently they can adjust it to achieve the expected objective.

Due to the limited abilities of the authors, there may be some errors when predicting grain output, however, in the next three years, China's annul grain output will be stabilized at about 600 million tons, which may grow slowly though.

\section{References}

Cheng, P., \& Yumin, P. (2011). Gas emission prediction with the RBF network optimized by PSO. Safety Science and Technology of China, 7(11), 77-81.

Deqing, W., Feifei, W., \& Wanchuang, Z. (2014). The prediction of non-stationary nonlinear time series based on the EMD technique. Systems Engineering, 32(5), 138-143.

Farge, M. W. (1992). Avelet transforms and their applications to turbulence. Annual Review of Fluid Mechanics, 395-457. https://doi.org/10.1146/annurev.fl.24.010192.002143

Guoxiang, L. (1999). The cycle fluctuation of grain production in China since 1949. Chinese Rural Observation, $5,44-51$.

Guoxiang, L., \& Jinsong, C. (2001). Food production and food security. Chinese Rural Economy, 4, 4-10.

He, S., \& Xiquan, S. (2000). Institutional change and the long wave of food production. Chinese Rural Observation, 37-46.

Hengguang, Z., \& Maoling, L. (2008). Chinese energy supply and demand analysis based on EMD and the dynamic prediction. Geography and Geographic Information Science, 24(3), 67-70.

Hengguang, Z., Zhenshan, L., \& Renjun, L. (2008). The dynamics prediction of bearing capacity of land in Shandong province based on EMD. Geographical Science, 28(2), 219-223.

Hongbin, L. (2009). The analysis of China's grain production fluctuation and its structure. Agricultural Technology Economy, 5, 91-96.

Hua, Y., \& Qiyuan, L. (2009). Speech recognition technology based on RBF neural network. Control Engineering, 16, 90-93.

Huiyu, L., Mingyang, Z., \& Zhenshan, L. (2004). Multiple time scale analysis of grain production in Jiangsu province Since1949. Resources Science, 26(1), 107-112.

Huiyu, L., Zhenshan, L., \& Mingyang, Z. (2005). The multi-scale analysis of the grain production fluctuation in China and causes based on EMD. Natural Resources, 20(5), 745-751.

Jie, Z., Lipeng, L., Penghui, J., Jinde, J., \& Xiaolei, Z. (2013). Multi-scale and associated dynamic analysis of grain production in Hubei province. Economic Geography, 33(7), 140-147.

Jieyong, W., \& Yansui, L. (n. d.). China's grain production pattern evolution and its driving mechanism from 1990 to 2005. Resources Science, 31(7), 1187-1194.

Ling, K. (2011). Grain output prediction based on the RBF neural network. Computer Simulation, 28(11), 189-200.

Lipeng, L., Jie, Z., Penghui, J., \& Shengwu, Z. (2013). Drive on the change of grain yield in Gansu province analysis and its trend prediction. Economic Geography, 33(4), 125-131.

Lipeng, L., Jie, Z., Shengwu, Z., \& Jinde, J. (2014). The multi-scale and correlation analysis of grain production 
in Gansu province. Arid Land Geography, 37(2), 397-405.

Lixiang, Z., Hui, P., \& Xingchu, J. (2008). Impact analysis of the amount of fertilizer at different stages to food production in China-based on the panel data of 30 provinces in 1952-2006. Agricultural Technology Economy, 4(7), 85-94.

Mei, Z. (1997). Grain production fluctuation analysis and the prediction of 1997. Chinese Rural Economy, 43-48.

Qingsong, L., Sujun, D., Guojin, X., Qian, W., \& Jiawei, Z. (2015). The fluctuation characteristics and influence factors of grain production in Henan province. Chinese Agriculture Notified, 31(18), 226-230.

Ruanlian, F., \& HongJie, B. (2012). The analysis of house prices cycle fluctuation based on empirical mode decomposition. Chinese Management Science, 20(3), 41-46.

Saili, X., Xiuyin, Z., Mengzhi, D., \& Hongwan, Y. (2014). The fluctuation analysis of grain production in Henan province based on HP filter. Journal of Henan Agricultural University, 48(6), 785-789.

Shutao, W., Xinwang, L., Mingxin, M., \& Hao, X. (2011). Research on the grain fluctuation influence factors in Hebei province based on improved grey correlation. Chinese Agricultural Science, 44(1), 176-184.

Suwen, Z. (2005). The analysis of grain production fluctuation and its trend in Henan province. Hunan Normal University, 2005.

Wenliang, Z., Junping, H., \& Zhen, H. (2012). Multi-scale analysis of the changes of grain production in Henan province. Journal of Henan University, 42(6), 732-772.

Xinwang, L., Shutao, W., \& Mingxin, M. (2009). The space-time multi-scale analysis of grain production fluctuation in Hebei province and causes based on EMD. Natural Resources, 29(11), 1994-2004.

Yan, S., Zhenshan, L., \& Huiyu, L. (2006). The breakthrough characteristics and driving mechanism of Chinese cultivated land quantity change. Resources Science, 28(5), 57-61.

Yingjun, C., \& Qinhua, C. (2013). The application of RBF neural network in the high speed milling surface roughness prediction. Combination Machine Tools and Automatic Processing Technology, 6(6), 6-8.

Yongjun, D., Weiqian, W., \& Chun, C. (2001). The boundary problem in EMD and Hilbert transformation. Chinese Science Bulletin, 46(3), 257-263.

Yubin, W., Junpeng, J., Xiaozhi, W., \& Huiping, C. (2007). The empirical analysis of affecting factors of Chinese grain production. Agronomy Journal of Beijing, 22(4), 38-41.

Zhenshan, L., \& Shuguang, W. (2004). The temperature analysis of northern hemisphere in almost four hundred years: Application of EMD method. Tropical Weather, 20(1), 90-96.

\section{Copyrights}

Copyright for this article is retained by the author(s), with first publication rights granted to the journal.

This is an open-access article distributed under the terms and conditions of the Creative Commons Attribution license (http://creativecommons.org/licenses/by/4.0/). 